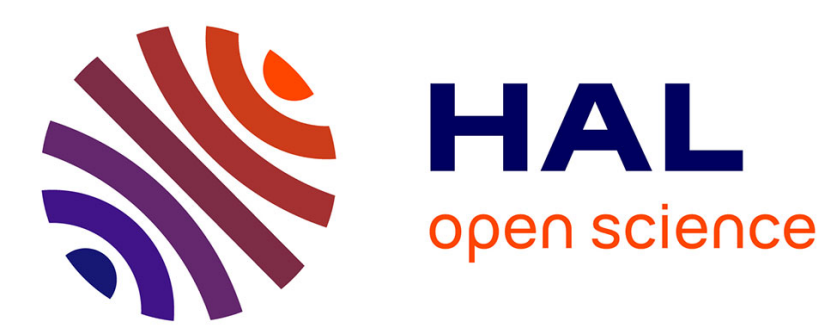

\title{
Error bounds for the reliability index in finite element reliability analysis
}

L. Gallimard

\section{To cite this version:}

L. Gallimard. Error bounds for the reliability index in finite element reliability analysis. International Journal for Numerical Methods in Engineering, 2011. hal-01689922

\section{HAL Id: hal-01689922 \\ https://hal.parisnanterre.fr/hal-01689922}

Submitted on 22 Jan 2018

HAL is a multi-disciplinary open access archive for the deposit and dissemination of scientific research documents, whether they are published or not. The documents may come from teaching and research institutions in France or abroad, or from public or private research centers.
L'archive ouverte pluridisciplinaire HAL, est destinée au dépôt et à la diffusion de documents scientifiques de niveau recherche, publiés ou non, émanant des établissements d'enseignement et de recherche français ou étrangers, des laboratoires publics ou privés. 


\title{
Error bounds for the reliability index in finite element reliability analysis
}

\author{
L. Gallimard ${ }^{*} \dagger$ \\ LEME, Université Paris Ouest Nanterre La Défense, 50 rue de Sèvres, 92410 Ville d’Avray, France
}

\begin{abstract}
SUMMARY
This work presents an extension of the goal-oriented error estimation techniques to the reliability analysis of a linear elastic structure. We use a first-order reliability method in conjunction with a finite element analysis (FEA) to compute the failure probability of the structure. In such a situation the output of interest that is computed from the FEA is the reliability index $\beta$. The accuracy of this output, and thus of the reliability analysis, depends, in particular, on the accuracy of the FEA. In this paper, upper and lower bounds of the reliability index are proposed, as well as simple bounds of the failure probability. An application to linear fracture mechanics is presented. Copyright (C) 2011 John Wiley \& Sons, Ltd.
\end{abstract}

KEY WORDS: $\quad$ structural reliability; finite element analysis; error bounds; goal-oriented error estimation; reliability index; FORM approximation

\section{INTRODUCTION}

Many applications in structural analysis require taking into account stochastic properties of material, geometry or loads. Different methods based on the probability theory have been developed to quantify the effect of data uncertainties. First-order reliability method (FORM) [1,2] is commonly used in reliability analysis. It is based on the computation of a reliability index $\beta$ [3], followed by an estimation of the failure probability. In many cases, the structural response cannot be expressed explicitly and the reliability analysis needs to be coupled with a finite element method. This coupling implies difficulties, such as prohibitive computational effort. Moreover, the development of a finite element discretized model starting from a continuous mechanical model leads to a partial loss of the information contained in the continuous model and, thus, to the introduction of discretization errors. As a consequence, the finite element analysis (FEA) introduces an approximation error in the computation of the reliability index $\beta$ that depends on the mesh used. The control of this discretization error is then necessary in order to minimize the computation effort, as well to assess the quality of the computed reliability index.

Methods have been developed over many years to evaluate the global quality of FEA [4-6]. For linear problems, all of these methods provide a global energy-based estimate of the discretization error. When the dimensioning criteria involve particular outputs of the FEA (stresses, displacements, intensity factors, etc.) this information is insufficient for dimensioning purposes. The development of error estimators for such quantities was initiated in the 1980s [7, 8]. Recently, numerous works have been published that provide error estimates and bounds for several quantities of interest

\footnotetext{
${ }^{*}$ Correspondence to: L. Gallimard, LEME, Université Paris Ouest Nanterre La Défense, 50 rue de Sèvres, 92410 Ville d'Avray, France.

†E-mail: laurent.gallimard@u-paris10.fr
} 
[9-16]. However, these works have been developed for deterministic FEA and little research has been carried out on the verification of probabilistic models [17, 18].

In this paper, we consider the case of a linear elastic structure where the limit-state function $G$ is written as: $G=I_{c}-I_{h}$, where $I_{c}$ is the resistance of the structure and $I_{h}$ is a particular output evaluated by the FEA. The calculation of the failure probability is performed by the FORM approximation. In such a computation three sources of errors are introduced:

- an algorithm error due to the gradient-based algorithm that is used to compute the reliability index,

- a geometric error due to the FORM approximation,

- a discretization error due to the computation of $I_{h}$ by an FEA.

As far we know, only the first two points have been addressed in the literature (see [19-22]). The objective of this work is to study the effect of the finite element discretization on the computation of the reliability index $\beta$ and to propose upper and lower bounds for this reliability index, which take into account the approximation errors introduced by the FEA.

The paper is organized as follows: In Section 2, we present the basis of the reliability analysis. In Section 3, we describe the problem to be solved. Section 4 is devoted to the presentation of a method to obtain bounds of the reliability index, as well as bounds of the FORM failure probability. Finally, Section 5 presents an application to linear fracture mechanics.

\section{PRINCIPLE OF RELIABILITY ANALYSIS}

In this section, we describe the problem of reliability assessment and we introduce the reliability index $[1,23,24]$. Structural reliability aims at computing the probability of failure of a mechanical system by accounting for uncertainties arising in a model description (geometry, material properties) or the environmental data (prescribed displacement and external forces).

Let $\mathbf{m}$ be the vector of the model parameters on which there are some uncertainties, and let $N$ be the size of $\mathbf{m}$. This vector is modelled by a random vector $\mathbf{X}$ and $f_{\mathbf{X}}(\mathbf{x})$ is the probability density function. The structure failure state is defined by a limit-state function $G$ such that

- $G(\mathbf{x})<0$ is a failure state for the structure,

- $G(\mathbf{x})=0$ is the limit state,

- $G(\mathbf{x})>0$ is a safe state for the structure.

The random variable defined by $Z=G(\mathbf{X})$ is called the safety margin of the structure. According to the definition of $G$, the system fails when this margin is lower or equal to zero. The failure probability $P_{f}$ is then given by:

$$
P_{f}=\int_{G(\mathbf{x}) \leqslant 0} f_{\mathbf{X}}(\mathbf{x}) \mathrm{d} \mathbf{x}
$$

The evaluation of the integral defined by Equation (1) is not easy because it represents a very small quantity and because the probability density function is not usually well known because of the scarcity of the statistical data. A classical way to evaluate this integral is to use the first- or the second-order reliability methods FORM/SORM [1]. They are based on the computation of a reliability index, followed by an estimation of the failure probability.

In [3], the authors propose to define the limit-state function $G$ in the space of independent standard Gaussian variables instead of the space of the physical variables. The transformation from the physical variables $\mathbf{X}$ to the normal variables $\mathbf{Y}$ is given by

$$
\mathbf{X}=T(\mathbf{Y})
$$


This transformation is called the isoprobabilistic transformation which transforms the probability integral into

$$
P_{f}=\int_{G(\mathbf{x}) \leqslant 0} f_{\mathbf{X}}(\mathbf{x}) \mathrm{d} \mathbf{x}=\int_{G(T(\mathbf{y})) \leqslant 0} \phi_{Y}(\mathbf{y}) \mathrm{d} \mathbf{y}
$$

where $\phi_{Y}(\mathbf{y})$ is the $n$-dimensional standard normal density with normal components. The limit-state function in the space of standard independent variables $\mathbf{Y}$ is written as:

$$
G(\mathbf{x})=G(T(\mathbf{y})) \equiv H(\mathbf{y})
$$

In this space, the Hasofer-Lind reliability index $\beta$, is defined as the minimum distance between the origin of the space $O$ and the surface of the limit state

$$
\beta=\min _{H(\mathbf{y}) \leqslant 0} \sqrt{\mathbf{y}^{\mathrm{T}} \mathbf{y}}
$$

The solution of this problem gives the value of $\beta$ as well as the coordinates $y_{i}^{*}$ of a point $P^{*}$ in the space called the design point. The FORM failure probability is given by

$$
P_{f, \mathrm{FORM}}=\Phi(-\beta)
$$

where $\Phi$ is the cumulative distribution function of a standard Gaussian variable.

\section{THE PROBLEM TO BE SOLVED}

\subsection{The elastic model}

Let us consider a $2 \mathrm{D}$ elastic structure defined in a domain $\Omega$ bounded by $\partial \Omega$. The external actions on the structure are represented by a prescribed displacement $\mathbf{u}_{d}$ over a subset $\Gamma_{D}$ of the boundary, a surface force density $\mathbf{T}$ defined over $\Gamma_{N}=\partial \Omega-\Gamma_{D}$, and a body force density $\mathbf{b}$ defined in $\Omega$. The Hooke's operator of the material is denoted by $\mathbf{K}$. Thus, the problem can be formulated as: Find a displacement field $\mathbf{u}$ and a stress field $\boldsymbol{\sigma}$ defined in $\Omega$ which verify:

- the kinematic constraints:

$$
\mathbf{u} \in \mathscr{U} \quad \text { and }\left.\quad \mathbf{u}\right|_{\Gamma_{D}}=\mathbf{u}_{d}
$$

- the equilibrium equations:

$$
\boldsymbol{\sigma} \in \mathscr{S} \quad \text { and } \quad \forall \mathbf{u}^{*} \in \mathscr{U}_{0}-\int_{\Omega} \boldsymbol{\sigma}: \varepsilon\left(\mathbf{u}^{*}\right) \mathrm{d} \Omega+\int_{\Omega} \mathbf{b} \cdot \mathbf{u}^{*} \mathrm{~d} \Omega+\int_{\Gamma_{N}} \mathbf{T} \cdot \mathbf{u}^{*} d \Gamma=0
$$

- the constitutive relation:

$$
\sigma=\mathbf{K} \varepsilon(\mathbf{u})
$$

$\mathscr{U}$ is the space in which the displacement field is being sought, $\mathscr{S}=\mathscr{L}_{2}[\Omega]^{3}$ the space of the stresses, $\mathscr{U}_{0}$ the space of the fields in $\mathscr{U}$ which are zero on $\Gamma_{D}$, and $\varepsilon(\mathbf{u})$ denotes the linearized deformation associated with the displacement:

$$
[\boldsymbol{\varepsilon}(\mathbf{u})]_{i j}=\frac{1}{2}\left(u_{i, j}+u_{j, i}\right)
$$

\subsection{Computation of the reliability index}

In a classical deterministic case, the objective of the computation is to verify that a quantity of interest denoted $I(\mathbf{u})$, depending on the solution of the mechanical problem $u$, is inferior to a critical value $I_{c}$. In a stochastic computation we assume that the data (prescribed displacement and forces, geometry) and the critical value $I_{c}$ are no longer deterministic and can be described by 
stochastic functions. The solution of the model problem becomes a stochastic displacement field denoted $u(\mathbf{X})$. The structure failure state is defined by a limit-state function $G(\mathbf{X})=I_{c}(\mathbf{X})-I(\mathbf{u}(\mathbf{X}))$ such that

- $I(\mathbf{u}(\mathbf{x}))>I_{c}(\mathbf{x})$ is a failure state for the structure,

- $I(\mathbf{u}(\mathbf{x}))=I_{c}(\mathbf{x})$ is the limit state,

- $I(\mathbf{u}(\mathbf{x}))<I_{c}(\mathbf{x})$ is a safe state for the structure.

The objective of the computation becomes to compute the probability of failure of the structure by using the FORM approximation, and the computation of the reliability index $\beta$. In the space of the independent standard Gaussian variables $\mathbf{Y}$, the failure domain is given by

$$
\mathscr{D}=\{\mathbf{y} ; H(\mathbf{y}) \leqslant 0\} \quad \text { with } H(\mathbf{y})=I_{c}(\mathbf{y})-I(\mathbf{u}(\mathbf{y}))
$$

and computing the reliability index consist in solving the following minimization problem: find $\beta$ such that

$$
\beta=\min _{\mathbf{y} \in \mathscr{D}} \sqrt{\mathbf{y}^{\mathrm{T}} \mathbf{y}}
$$

\subsection{Finite element analysis and approximation errors}

The Galerkin finite element method provides an approximation $\mathbf{u}_{h}(\mathbf{Y})$ to $\mathbf{u}(\mathbf{Y})$ in a finite element space $\mathscr{U}^{h} \subset \mathscr{U}$. The finite element approximation transforms the failure domain

$$
\mathscr{D}_{h}=\left\{\mathbf{y} ; H_{h}(\mathbf{y}) \leqslant 0\right\} \quad \text { with } H_{h}(\mathbf{y})=I_{c}(\mathbf{y})-I\left(\mathbf{u}_{h}(\mathbf{y})\right)
$$

and the minimization problem becomes: find $\beta_{h}$ such that

$$
\beta_{h}=\min _{\mathbf{y} \in \mathscr{D}_{h}} \sqrt{\mathbf{y}^{\mathrm{T}} \mathbf{y}}
$$

Two errors are introduced in the computation of the reliability index

- an error due to the resolution of the optimization problem under constraint (14),

- an error due to the finite element discretization.

In this paper, we focus on the error introduced by the FEA and we assume that the optimization problem is solved exactly. The optimization problem described in Figure 1 is solved by an improved

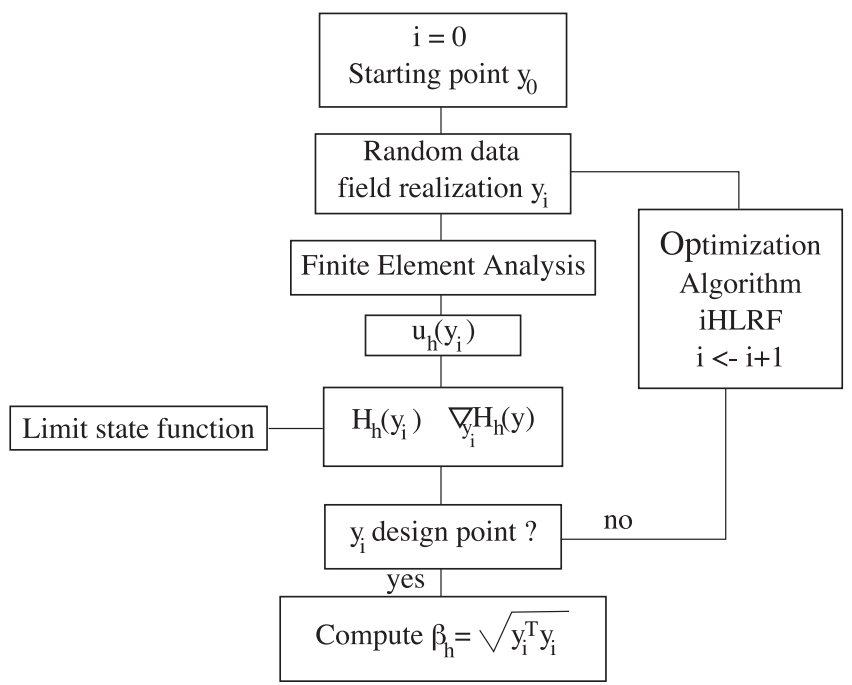

Figure 1. Computation of the reliability index. 
version of the classical HLRF algorithm [25], the iHLRF algorithm developed in [19, 26, 27] (see $[21,26,27]$ for the detailed studies on the convergence of the optimization problem).

\section{BOUNDS OF THE RELIABILITY INDEX}

In this section, upper and lower bounds for the reliability index are presented. These bounds allow computing the bounds of the failure probability obtained by the FORM method. The computation of these bounds assume that the quantity of interest $I(\mathbf{u})$ is a linear functional.

\subsection{Bounds of the reliability index}

Let us assume that we are able to compute lower and upper bounds of the quantity of interest $I(\mathbf{u}(\mathbf{y}))$, from the finite element solution and the data, such that

$$
I^{-}\left(\mathbf{u}_{h}(\mathbf{y})\right) \leqslant I(\mathbf{u}(\mathbf{y})) \leqslant I^{+}\left(\mathbf{u}_{h}(\mathbf{y})\right)
$$

We define a reliability index $\beta_{h}^{\text {low }}$ associated with the upper bound $I^{+}\left(\mathbf{u}_{h}(\mathbf{y})\right)$

$$
\beta_{h}^{\text {low }}=\min _{\mathbf{y} \in \mathscr{D}_{h}^{+}} \sqrt{\mathbf{y}^{\mathrm{T}} \mathbf{y}}
$$

where $\mathscr{D}_{h}^{+}$is the failure domain defined by

$$
\mathscr{D}_{h}^{+}=\left\{\mathbf{y} ; H_{h}^{+}(\mathbf{y})=I_{c}(\mathbf{y})-I^{+}\left(\mathbf{u}_{h}(\mathbf{y})\right) \leqslant 0\right\}
$$

and a reliability index $\beta_{h}^{\text {upp }}$ associated with the lower bound $I^{-}\left(\mathbf{u}_{h}(\mathbf{y})\right)$

$$
\beta_{h}^{\text {upp }}=\min _{\mathbf{y} \in \mathscr{D}_{h}^{-}} \sqrt{\mathbf{y}^{\mathrm{T}} \mathbf{y}}
$$

where $\mathscr{D}_{h}^{-}$is the failure domain defined by

$$
\mathscr{D}_{h}^{-}=\left\{\mathbf{y} ; H_{h}^{-}(\mathbf{y})=I_{c}(\mathbf{y})-I^{-}\left(\mathbf{u}_{h}(\mathbf{y})\right) \leqslant 0\right\}
$$

Equation (15) leads to

$$
I^{c}(\mathbf{y})-I^{+}\left(\mathbf{u}_{h}(\mathbf{y})\right) \leqslant I^{c}(\mathbf{y})-I(\mathbf{u}(\mathbf{y})) \leqslant I^{c}(\mathbf{y})-I^{-}\left(\mathbf{u}_{h}(\mathbf{y})\right)
$$

and hence to

$$
\mathscr{D}_{h}^{-} \subset \mathscr{D} \subset \mathscr{D}_{h}^{+}
$$

From (21) we deduce the following bounds for the reliability index $\beta$ :

$$
\beta_{h}^{\text {low }} \leqslant \beta \leqslant \beta_{h}^{\text {upp }}
$$

Let us define the relative error on the reliability index

$$
\varepsilon\left(\beta_{h}\right)=\frac{\beta-\beta_{h}}{\beta_{h}}
$$

Equation (22) leads to the following error bounds:

$$
\frac{\beta_{h}^{\text {low }}}{\beta_{h}}-1 \leqslant \varepsilon\left(\beta_{h}\right) \leqslant \frac{\beta_{h}^{\text {upp }}}{\beta_{h}}-1
$$

An estimation of this error is computed by

$$
\hat{\varepsilon}\left(\beta_{h}\right)=\max \left(\left|\frac{\beta_{h}^{\text {low }}}{\beta_{h}}-1\right|,\left|\frac{\beta_{h}^{\text {upp }}}{\beta_{h}}-1\right|\right)
$$




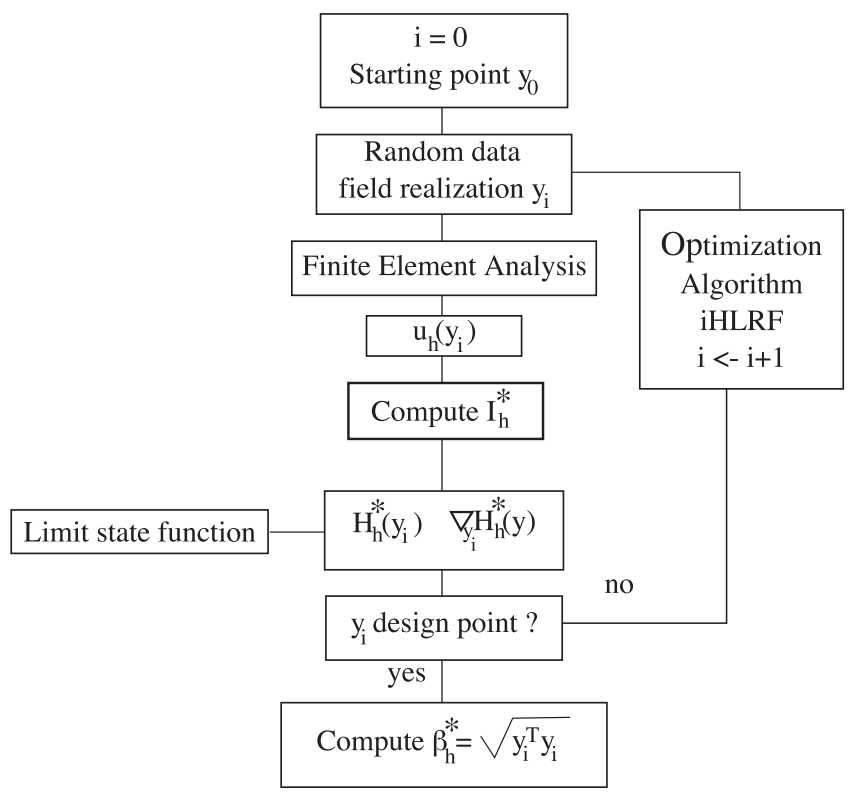

Figure 2. Computation of the bounds of the reliability index: $*=+$ or $*=-$.

As $\Phi(-\beta)$ is a monotonous function, the computation of lower and upper bounds for the reliability index leads to simple bounds of the FORM failure probability due to the finite element approximation

$$
P_{f, \mathrm{FORM}}^{\text {low }} \leqslant P_{f, \mathrm{FORM}} \leqslant P_{f, \mathrm{FORM}}^{\mathrm{upp}}
$$

with $P_{f, \mathrm{FORM}}^{\text {low }}=\Phi\left(-\beta_{h}^{\text {upp }}\right), P_{f, \mathrm{FORM}}^{\text {upp }}=\Phi\left(-\beta_{h}^{\text {low }}\right)$ and $P_{f, \mathrm{FORM}}=\Phi(-\beta)$.

The bounds $\beta_{h}^{\text {low }}$ and $\beta_{h}^{\text {upp }}$ are computed by solving successively two minimization problems: a minimization problem $\mathscr{M}^{+}$related to the upper bound of the quantity of interest, and a minimization problem $\mathscr{M}^{-}$related to the lower bound of the quantity of interest. These minimization problems are solved by the iHLRF algorithm [19]. At each step, the optimization algorithm requires the construction of an upper bound of the quantity of interest $I^{+}\left(\mathbf{u}_{h}(\mathbf{y})\right.$ ) (or a lower bound $\left.I^{-}\left(\mathbf{u}_{h}(\mathbf{y})\right)\right)$, as described in Figure 2. These quantities can be related to the bounds of the discretization error in the quantity of interest. Let us introduce $\Delta_{h}^{+}(\mathbf{y})$ and $\Delta_{h}^{-}(\mathbf{y})$ such that

$$
\begin{aligned}
\Delta_{h}^{-}(\mathbf{y}) & =I\left(\mathbf{u}_{h}(\mathbf{y})\right)-I^{-}\left(\mathbf{u}_{h}(\mathbf{y})\right) \\
\Delta_{h}^{+}(\mathbf{y}) & =I^{+}\left(\mathbf{u}_{h}(\mathbf{y})\right)-I\left(\mathbf{u}_{h}(\mathbf{y})\right)
\end{aligned}
$$

By introducing $\Delta_{h}^{-}(\mathbf{y})$ and $\Delta_{h}^{+}(\mathbf{y})$ in Equation (15), one obtains

$$
-\Delta_{h}^{-}(\mathbf{y}) \leqslant I(\mathbf{u}(\mathbf{y}))-I\left(\mathbf{u}_{h}(\mathbf{y})\right) \leqslant+\Delta_{h}^{+}(\mathbf{y})
$$

where $I(\mathbf{u}(\mathbf{y}))-I\left(\mathbf{u}_{h}(\mathbf{y})\right)$ is the discretization error in the quantity of interest. The following section is devoted to the computation of the bounds $\Delta_{h}^{-}(\mathbf{y})$ and $\Delta_{h}^{+}(\mathbf{y})$.

\subsection{Estimation of the outputs of interest}

In this section, we briefly recall the techniques developed in the literature for the case where the quantity of interest is a linear functional $I$ of the displacement

$$
I(\mathbf{u}(\mathbf{y}))=\oint_{\Omega} \boldsymbol{\Sigma}: \boldsymbol{\varepsilon}(\mathbf{u}(\mathbf{y})) \mathrm{d} \Omega+\int_{\Omega} \mathbf{f} \cdot \mathbf{u}(\mathbf{y}) \mathrm{d} \Omega
$$


where $\boldsymbol{\Sigma}$ and $\mathbf{f}$ are the given extractors which define the output of interest (an example of the output of interest is given in Section 5). We refer the reader to References [11-14] for a detailed description of this approach, and to [16, 28-31] for the extension to non-linear quantities of interest. For the sake of clarity we will omit the reference to the space of the random parameters $\mathbf{Y}$. Let us introduce the classical discretization error in Equation (28)

$$
\mathbf{e}_{h}=\mathbf{u}-\mathbf{u}_{h}
$$

due to the linearity assumption, one has

$$
I\left(\mathbf{e}_{h}\right)=I(\mathbf{u})-I\left(\mathbf{u}_{h}\right)
$$

thus, the estimate of $\left|I(\mathbf{u})-I\left(\mathbf{u}_{h}\right)\right|$ is equivalent to the estimate of $\left|I\left(\mathbf{e}_{h}\right)\right|$. Following [12], we consider the following auxiliary problem: find $\mathbf{u}^{\text {aux }} \in \mathscr{U}_{0}$ and $\sigma^{\text {aux }}=\mathbf{K} \varepsilon\left(\mathbf{u}^{\text {aux }}\right)$ such that:

$$
\int_{\Omega} \mathbf{K} \boldsymbol{\varepsilon}\left(\mathbf{u}^{*}\right): \boldsymbol{\varepsilon}\left(\mathbf{u}^{\text {aux }}\right) \mathrm{d} \Omega=I\left(\mathbf{u}^{*}\right) \quad \forall \mathbf{u}^{*} \in \mathscr{U}_{0}
$$

One obtains the following relation for the error estimate:

$$
I(\mathbf{u})-I\left(\mathbf{u}_{h}\right)=I\left(\mathbf{e}_{h}\right)=\int_{\Omega} \mathbf{K} \boldsymbol{\varepsilon}\left(\mathbf{e}_{h}\right): \varepsilon\left(\mathbf{e}_{h}^{\text {aux }}\right) \mathrm{d} \Omega
$$

where $\mathbf{e}_{h}^{\text {aux }}=\mathbf{u}^{\text {aux }}-\mathbf{u}_{h}^{\text {aux }}$ is the discretization error of the auxiliary problem.

Following [14], the error in the quantity of interest can be written as

$$
\begin{aligned}
I(\mathbf{u})-I\left(\mathbf{u}_{h}\right) & =\int_{\Omega} \mathbf{K} \varepsilon\left(\mathbf{e}_{h}\right): \varepsilon\left(\mathbf{e}_{h}^{\mathrm{aux}}\right) \mathrm{d} \Omega \\
& =\frac{1}{4}\left\|\mathbf{u}^{+}-\mathbf{u}_{h}^{+}\right\|_{u}^{2}-\frac{1}{4}\left\|\mathbf{u}^{-}-\mathbf{u}_{h}^{-}\right\|_{u}^{2}
\end{aligned}
$$

where $\|\mathbf{u}\|_{u}=\int_{\Omega} \mathbf{K} \boldsymbol{\varepsilon}(\mathbf{u}): \varepsilon(\mathbf{u}) \mathrm{d} \Omega, \mathbf{u}^{ \pm}=s \mathbf{u} \pm s^{-1} \mathbf{u}^{\text {aux }}$ and $\mathbf{u}_{h}^{ \pm}=s \mathbf{u}_{h} \pm s^{-1} \mathbf{u}_{h}^{\text {aux }}$. The scaling factor $s$ is chosen so that $\left\|s \mathbf{e}_{h}\right\|_{u}$ and $\left\|s^{-1} \mathbf{e}_{h}^{\text {aux }}\right\|_{u}$ have the same amplitude

$$
s=\sqrt{\left\|\mathbf{e}_{h}^{\mathrm{aux}}\right\|_{u} /\left\|\mathbf{e}_{h}\right\|_{u}}
$$

In order to determine bounds for the error in the quantity of interest, Equation (34) indicates that it is sufficient to bound the energy norm of $\mathbf{u}^{+}-\mathbf{u}_{h}^{+}$and $\mathbf{u}^{-}-\mathbf{u}_{h}^{-}$. Methods to obtain bounds on the error in the energy norm are now available in the literature [9-11]. In this paper, the methodology proposed in [32] which is based on the concept of constitutive relation error estimator [5] is used to build bounds of these errors. This method leads to lower bounds $\tilde{e}_{h}^{ \pm}$and upper bounds $\hat{e}_{h}^{ \pm}$such that (we refer the reader to [32] for more details)

$$
\begin{aligned}
& \tilde{e}_{h}^{+} \leqslant\left\|\mathbf{u}^{+}-\mathbf{u}_{h}^{+}\right\|_{u} \leqslant \hat{e}_{h}^{+} \\
& \tilde{e}_{h}^{-} \leqslant\left\|\mathbf{u}^{-}-\mathbf{u}_{h}^{-}\right\|_{u} \leqslant \hat{e}_{h}^{-}
\end{aligned}
$$

Once these bounds are computed, the classical upper and lower bounds are easily derived from Equations (34) and (35)

$$
-\Delta_{h}^{-} \leqslant I(\mathbf{u})-I\left(\mathbf{u}_{h}\right) \leqslant \Delta_{h}^{+}
$$

where

$$
\Delta_{h}^{-}=\frac{1}{4}\left(\hat{e}_{h}^{-}\right)^{2}-\frac{1}{4}\left(\tilde{e}_{h}^{+}\right)^{2} \quad \text { and } \quad \Delta_{h}^{+}=\frac{1}{4}\left(\hat{e}_{h}^{+}\right)^{2}-\frac{1}{4}\left(\tilde{e}_{h}^{-}\right)^{2}
$$

The bounds of the output of interest are directly computed from $I_{h}, \tilde{e}_{h}^{ \pm}, \hat{e}_{h}^{ \pm}$

$$
I^{+}\left(\mathbf{u}_{h}\right)=I\left(\mathbf{u}_{h}\right)+\frac{1}{4}\left(\hat{e}_{h}^{+}\right)^{2}-\frac{1}{4}\left(\tilde{e}_{h}^{-}\right)^{2} \quad \text { and } \quad I^{-}\left(\mathbf{u}_{h}\right)=I\left(\mathbf{u}_{h}\right)-\frac{1}{4}\left(\hat{e}_{h}^{-}\right)^{2}+\frac{1}{4}\left(\tilde{e}_{h}^{+}\right)^{2}
$$




\section{APPLICATION TO FRACTURE MECHANIC}

The output of interest $I$ in the following problems is the mode I stress intensity factor (SIF) $K_{I}$ computed at the crack tip in a cracked structure represented in Figure 3. An extraction operator is used to compute the SIF $K_{I}$. This extraction operator is based on a modification of Stern's contour integral [33] proposed in [15]. The calculation of the SIF consists in an integral around an arbitrary crown $\omega_{2}$ around the tip of the crack (Figure 4), and leads to $K_{I}$ without introducing any errors except for those due to the calculation of $u$

$$
K_{I}=I(\mathbf{u})=\int_{\omega_{2}}\left(\mathbf{K} \boldsymbol{\varepsilon}\left(\phi \tilde{\mathbf{u}}^{I}\right)-\phi \tilde{\boldsymbol{\sigma}}^{I}\right): \boldsymbol{\varepsilon}(\mathbf{u}) \mathrm{d} \omega-\int_{\omega_{2}} \tilde{\boldsymbol{\sigma}}^{I} \operatorname{grad}(\phi) \cdot \mathbf{u} \mathrm{d} \omega
$$

where $\tilde{\boldsymbol{\sigma}}^{I}$ and $\tilde{\mathbf{u}}^{I}$ are singular analytical solutions computed at the crack tip and $\phi$ a function such that $\phi(M)=1$ on $C_{1}$ and $\phi(M)=0$ on $C_{2}$ (for further details see [15, 33]).

The approximate value computed by an FEA $I_{h}$ will be denoted $K_{I h}$ with

$$
K_{I h}=I\left(\mathbf{u}_{h}\right)=\int_{\omega_{2}}\left(\mathbf{K} \boldsymbol{\varepsilon}\left(\phi \tilde{\mathbf{u}}_{h}^{I}\right)-\phi \tilde{\boldsymbol{\sigma}}^{I}\right): \boldsymbol{\varepsilon}\left(\mathbf{u}_{h}\right) \mathrm{d} \omega-\int_{\omega_{2}} \tilde{\boldsymbol{\sigma}}^{I} \operatorname{grad}(\phi) \cdot \mathbf{u}_{h} \mathrm{~d} \omega
$$

The limit state of the structure is given by a failure criterion which is the risk that the SIF $K_{I}$ reaches the toughness of the material $K_{I c}$

$$
G=K_{I c}-K_{I} \leqslant 0
$$

The studied structures are plates in plane stress with a finite crack shown in Figures 5 and 6. The deterministic parameters of the model are the Young modulus $E=210000 \mathrm{MPa}$, the Poisson ratio $v=0.25, L=32 \mathrm{~mm}, w=8 \mathrm{~mm}$. The random variables of the model are the crack length $a$, the toughness of the material $K_{I c}$ in the two examples, the loading $t_{1}$ in example 1 , the loading $t_{2}$ and the angle of the crack $\theta$ in example 2 ,

- the crack length $a$ is supposed to have a lognormal distribution with mean $m_{a}=2 \mathrm{~mm}$ and a standard deviation $\sigma_{a}=0.4$,

- the material toughness $K_{I c}$ is supposed to follow a normal distribution with a mean value of $m_{K}=4 \mathrm{MPamm}^{1 / 2}$ and a standard deviation of $\sigma_{K}=0.4$,

- the tensile load $t_{1}$ is supposed to follow a normal distribution with a mean value of $m_{t_{1}}=1 \mathrm{Mpa}$ and a standard deviation of $\sigma_{t_{1}}=0.1$,

- the shear load $t_{2}$ is supposed to follow a normal distribution with a mean value of $m_{t_{2}}=0.5 \mathrm{Mpa}$ and a standard deviation of $\sigma_{t_{2}}=0.1$,

- the angle of the crack $\theta$ is supposed to follow a normal distribution with a mean value of $m_{\theta}=5^{\circ}$ and a standard deviation of $\sigma_{\theta}=5^{\circ}$.

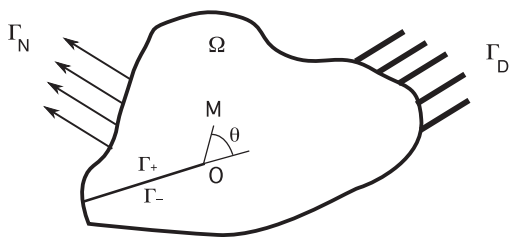

Figure 3. The structure $\Omega$.

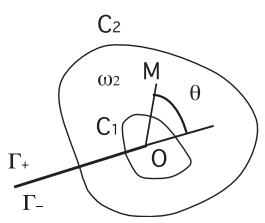

Figure 4. Zoom around the crack's tip. 


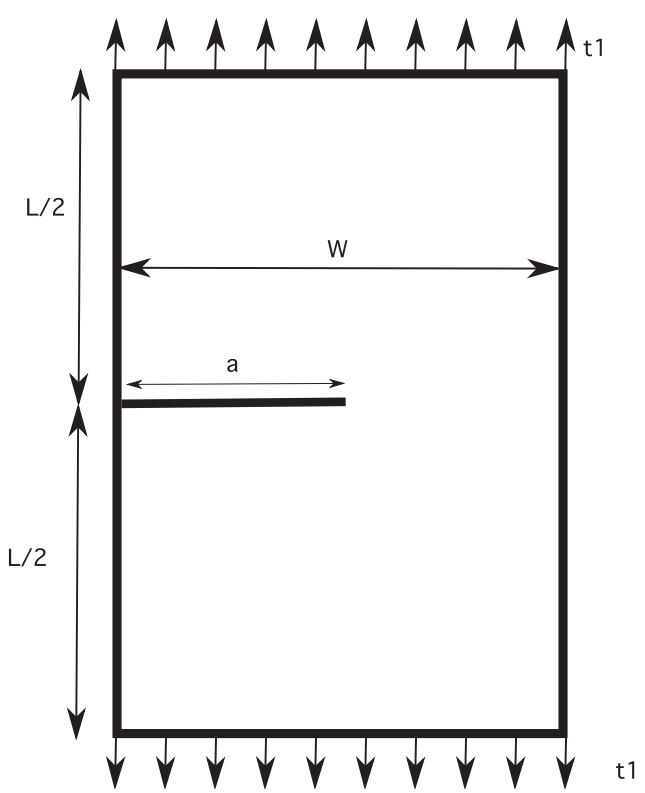

Figure 5. Example 1 (tensile load).

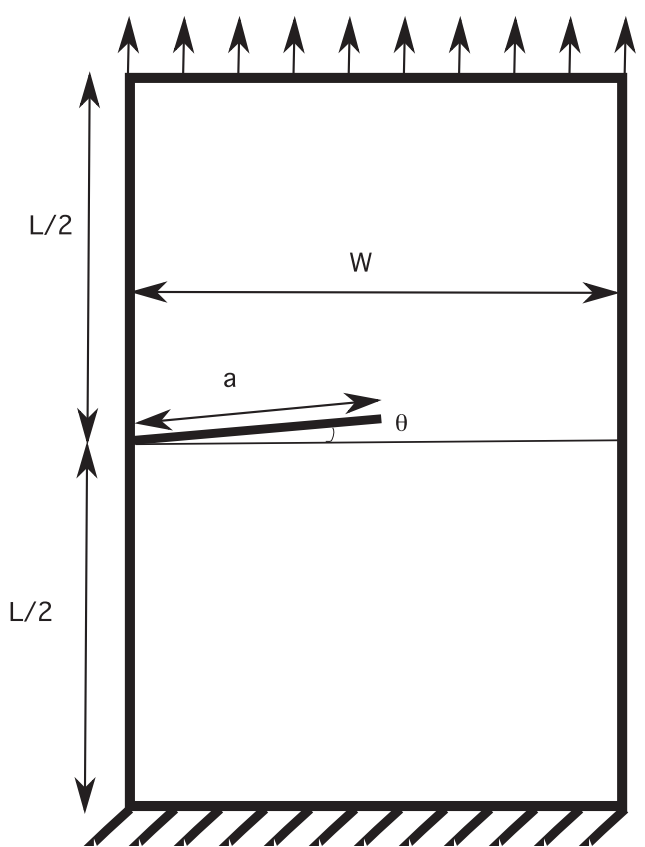

Figure 6. Example 2 (shear load).

These variables are easily transformed in standard normal variables $u_{a}, u_{t}, u_{K}$ and $u_{\theta}$

- $a=\exp \left(\lambda_{a} u_{a}+\mu_{a}\right)$,

- $t=\sigma_{t} u_{t}+m_{t}$,

- $K_{I c}=\sigma_{K} u_{K}+m_{K}$,

- $\theta=\sigma_{\theta} u_{\theta}+m_{\theta}$

where $\lambda_{a}$ and $\mu_{a}$ are the parameters of the lognormal distribution given by $\mu_{a}=\log \left(m_{a} /\right.$ $\left.\sqrt{1+\left(m_{a} / \sigma_{a}\right)^{2}}\right)=0.674$ and $\lambda_{a}=\sqrt{\log \left(1+\left(m_{a} / \sigma_{a}\right)^{2}\right)}=0.198$. The informations on the reliability models are summarized in Table I. 
Table I. Reliability models.

\begin{tabular}{lcc}
\hline & Example 1 & Example 2 \\
\hline $\mathbf{X}$ & $\left(a, t_{1}, K_{I c}\right)$ & $\left(a, \theta, t_{2}, K_{I c}\right)$ \\
$G$ & $K_{I c}-K_{I}\left(a, t_{1}\right)$ & $K_{I c}-K_{I}\left(a, \theta, t_{2}\right)$ \\
$\mathbf{Y}$ & $\left(u_{a}, u_{t}, u_{K}\right)$ & $\left(u_{a}, u_{\theta}, u_{t}, u_{K}\right)$ \\
$H$ & $\sigma_{K} u_{K}+m_{K}$ & $\sigma_{K} u_{K}+m_{K}$ \\
& $-K_{I}\left(\exp \left(\lambda_{a} u_{a}+\mu_{a}\right), \sigma_{t} u_{t}+m_{t}\right)$ & $-K_{I}\left(\exp \left(\lambda_{a} u_{a}+\mu_{a}\right), \sigma_{\theta} u_{\theta}+m_{\theta}, \sigma_{t} u_{t}+m_{t}\right)$ \\
\hline
\end{tabular}
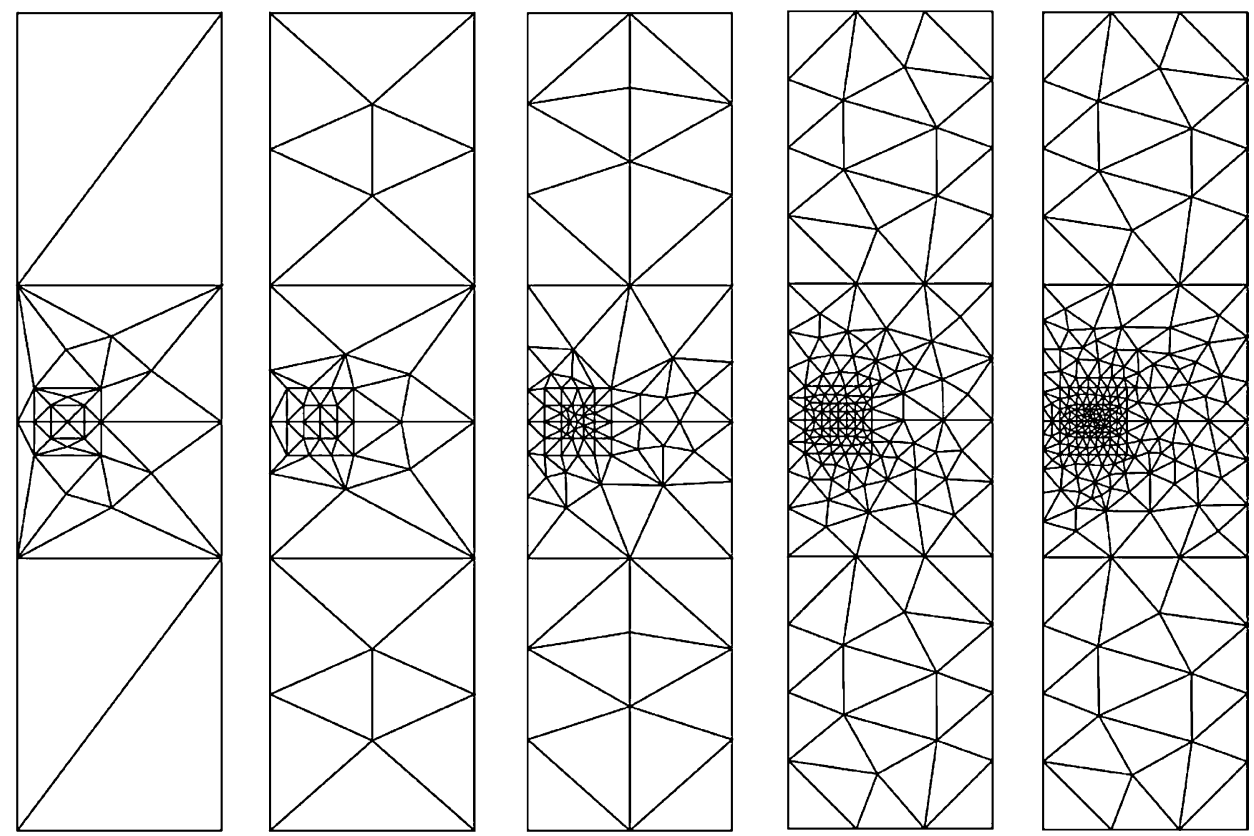

GIBI FECIT

Figure 7. Initial meshes (DoF: 234, 326, 670, 1250, 1846).

We use 6-node triangular elements to perform the finite element computations, and we study the evolution of the results as a function of the mesh size. The five meshes used are represented in Figure 7.

\section{Remark}

It must be noticed that as we need to remesh the structure on each iterations of the iHLRF algorithm where we modify the crack length or its angle, we use in fact different meshes during the computation of $\beta_{h}$. On the examples studied, the number of elements of these different meshes varies little (less than 10\%). We choose, as a reference for the number of degree of freedom, the mesh built with $a$ equal to its mean value and $\theta$ equal to 0 .

\subsection{Example 1: plate submitted to a tensile load}

For this problem, analytical results are available for $K_{I}$ in [34] (Equation (41)) with less than $0.1 \%$ error:

$$
K_{I}=\left(1 .-0.025 \alpha^{2}+0.06 \alpha^{4}\right) \sqrt{\sec \frac{\pi \alpha}{2}} t \sqrt{\pi a}
$$

where $\alpha=a / w$. 
Table II. Example 1 (tensile load): Deterministic computation $K_{I, \text { ref }}=2.575$.

\begin{tabular}{lccc}
\hline DoF & $K_{I h}$ & $K_{I h}^{+}$ & $K_{I h}^{-}$ \\
\hline 234 & 2.509 & 2.613 & 2.509 \\
326 & 2.522 & 2.592 & 2.540 \\
670 & 2.560 & 2.581 & 2.566 \\
1250 & 2.567 & 2.577 & 2.570 \\
1846 & 2.570 & 2.577 & 2.572 \\
\hline
\end{tabular}

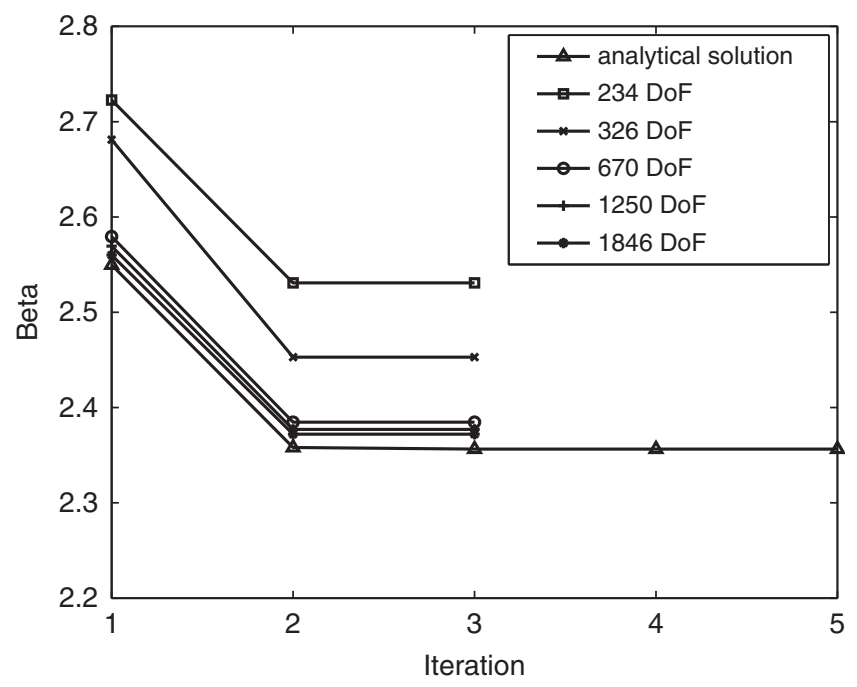

Figure 8. Convergence of the reliability index evaluation by an iHLRF algorithm (tensile load).

Table III. Example 1 (tensile load): Reliability computation $\left(\beta_{\text {ref }}=2.356\right)$.

\begin{tabular}{lcllcrrr}
\hline DoF & $\beta_{h}$ & $\beta_{h}^{\text {upp }}$ & $\beta_{h}^{\text {low }}$ & $\beta_{h}^{\text {upp }}-\beta_{h}^{\text {low }}$ & $\left|\varepsilon\left(\beta_{h}\right)\right|(\%)$ & $\hat{\varepsilon}\left(\beta_{h}\right)(\%)$ & $\rho$ \\
\hline 234 & 2.531 & 2.510 & 2.238 & 0.272 & 6.89 & 11.58 & 1.68 \\
326 & 2.453 & 2.417 & 2.326 & 0.091 & 3.93 & 5.18 & 1.32 \\
670 & 2.385 & 2.373 & 2.345 & 0.028 & 1.19 & 1.66 & 1.40 \\
1250 & 2.377 & 2.366 & 2.349 & 0.017 & 0.87 & 1.19 & 1.37 \\
1846 & 2.372 & 2.363 & 2.350 & 0.013 & 0.47 & 0.74 & 1.56 \\
\hline
\end{tabular}

We perform first a deterministic computation with the random variables set to their mean values. Equation (41) leads to a reference value for the SIF $K_{I \text {,ref }}=2.575$. The evolution of the FE SIF as well as the computed lower bound $K_{I h}^{-}$and upper bound $K_{I h}^{+}$are reported in Table II. It is worth noting that the deterministic computation gives SIFs that are smaller than the critical value. In this example, the computed bounds are very sharp, the lower bound and the upper bound are a better approximation of the reference value than the finite element solution. The fact that the finite element solution is outside the bounds is not inconsistent, as the relation (36) only implies that $K_{I h}^{-}$and $K_{I h}^{+}$bound the reference solution, but not necessarily the FE solution.

For the reliability computations, the initial values of the standard normal variables $u_{a}, u_{t}$ and $u_{K}$ are set to zero. We can obtain a reference value for $\beta$, using the iHLRF algorithm with $K_{I}$ computed from Equation (41). At convergence the reliability index is $\beta_{\text {ref }}=2.356$, and we will use this value as a reference value. The convergence of the iHLRF algorithm is shown in Figure 8 for the five meshes and for the analytical solution. It can be seen that for this simple problem the algorithm converges very rapidly.

The bounds of the reliability index as a function of the mesh size are reported in Table III. In this example, the gap between the computed bounds $\beta_{h}^{\text {upp }}$ and $\beta_{h}^{\text {low }}$ decreases rapidly. It must be 
Table IV. Example 1 (tensile load): Failure probability bounds $-P_{f, \text { ref }}=9.2 \times 10^{-3}$.

\begin{tabular}{lccc}
\hline DoF & $P_{f, h}$ & $P_{f, h}^{\text {low }}$ & $P_{f, h}^{\text {upp }}$ \\
\hline 234 & $5.7 \times 10^{-3}$ & $6.0 \times 10^{-3}$ & $1.26 \times 10^{-2}$ \\
326 & $7.1 \times 10^{-3}$ & $7.8 \times 10^{-3}$ & $1.00 \times 10^{-2}$ \\
670 & $8.5 \times 10^{-3}$ & $8.8 \times 10^{-3}$ & $9.5 \times 10^{-3}$ \\
1250 & $8.7 \times 10^{-3}$ & $9.0 \times 10^{-3}$ & $9.4 \times 10^{-3}$ \\
1846 & $8.8 \times 10^{-3}$ & $9.1 \times 10^{-3}$ & $9.4 \times 10^{-3}$ \\
\hline
\end{tabular}

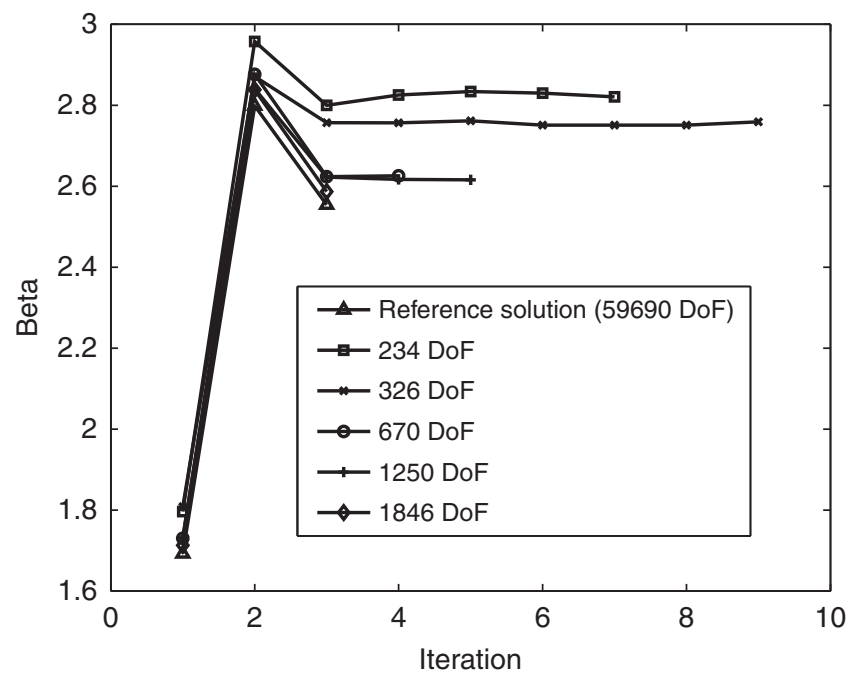

Figure 9. Convergence of the reliability index evaluation by an iHLRF algorithm (inclined crack).

noticed that, as in the deterministic computation of the bounds of $K_{I}$, the finite element evaluation of $\beta$ may be outside the computed bounds. However, $\beta_{\text {ref }}$ is inside the bounds $\beta_{h}^{\text {low }}$ and $\beta_{h}^{\text {upp }}$. The computation of the relative error estimate $\hat{\varepsilon}\left(\beta_{h}\right)$ (25) gives a valuable information on the accuracy of the reliability computation. It can be compared with the reference error on $\beta_{h}:\left|\varepsilon\left(\beta_{h}\right)\right|$ (Equation (23)), by computing an effectivity index $\rho=\hat{\varepsilon}\left(\beta_{h}\right) /\left|\varepsilon\left(\beta_{h}\right)\right|$. This effectivity index seems to be sufficiently sharp with values ranging from 1.32 to 1.68 .

Following Equation (6), the reference failure probability obtained with a FORM approximation is $P_{f \text {,ref }}=\Phi\left(-\beta_{\text {ref }}\right)=9.2 \times 10^{-3}$. The failure bounds derived from Equation (26) are presented in Table IV. It can be observed that, although the finite element probability of failure converge to the reference failure probability, the failure probability computed on the coarsest meshes is clearly inferior to the reference value and can lead to an underestimation of the risk. On the other hand, the computation of the bounds gives a good estimation of the failure probability on the third mesh (670 DoF) with an upper bound equal to $P_{f, h}^{\mathrm{upp}}=9.5 \times 10^{-3}$.

\subsection{Example 2: plate with an inclined crack}

The loading of the second problem involves a combination of modes I and II at the tip of the crack. We have computed a reference solution using the iHLRF algorithm with a refined mesh (59690 Dof). In this case we obtain $\beta_{\text {ref }}=2.548$. The convergence of the iHLRF algorithm is shown in Figure 9 for the five meshes and for the reference solution. The iHLRF algorithm converges rapidly when the mesh is sufficiently refined.

In Table V, we study the evolution of the bounds of the reliability index as a function of the mesh. Although the gap $\beta_{h}^{\text {upp }}-\beta_{h}^{\text {low }}$ is greater than in Example 1, the bounds become very accurate as soon as the mesh is sufficiently refined (less than three percent of error for the $670 \mathrm{DoF}$ mesh). 
Table V. Example 2 (inclined crack): Reliability computation $\left(\beta_{\text {ref }}=2.548\right)$.

\begin{tabular}{lcccccrr}
\hline DoF & $\beta_{h}$ & $\beta_{h}^{\text {upp }}$ & $\beta_{h}^{\text {low }}$ & $\beta_{h}^{\text {upp }}-\beta_{h}^{\text {low }}$ & $\left|\varepsilon\left(\beta_{h}\right)\right|(\%)$ & $\hat{\varepsilon}\left(\beta_{h}\right)(\%)$ & $\rho$ \\
\hline 234 & 2.821 & 2.723 & 2.485 & 0.238 & 9.68 & 11.91 & 1.23 \\
326 & 2.759 & 2.650 & 2.538 & 0.112 & 7.65 & 8.01 & 1.05 \\
670 & 2.626 & 2.586 & 2.541 & 0.045 & 2.97 & 3.24 & 1.09 \\
1250 & 2.616 & 2.586 & 2.545 & 0.041 & 2.60 & 2.71 & 1.04 \\
1846 & 2.587 & 2.567 & 2.539 & 0.030 & 1.51 & 1.86 & 1.23 \\
\hline
\end{tabular}

Table VI. Example 2 (inclined crack): Failure probability bounds- $P_{f, \text { ref }}=5.5 \times 10^{-3}$.

\begin{tabular}{lccc}
\hline DoF & $P_{f, h}$ & $P_{f, h}^{\text {low }}$ & $P_{f, h}^{\text {upp }}$ \\
\hline 234 & $2.4 \times 10^{-3}$ & $3.2 \times 10^{-3}$ & $6.5 \times 10^{-3}$ \\
326 & $2.9 \times 10^{-3}$ & $4.0 \times 10^{-3}$ & $5.6 \times 10^{-3}$ \\
670 & $4.3 \times 10^{-3}$ & $4.9 \times 10^{-3}$ & $5.5 \times 10^{-3}$ \\
1250 & $4.4 \times 10^{-3}$ & $4.9 \times 10^{-3}$ & $5.5 \times 10^{-3}$ \\
1846 & $5.1 \times 10^{-3}$ & $5.1 \times 10^{-3}$ & $5.6 \times 10^{-3}$ \\
\hline
\end{tabular}

The computation of the error estimate $\hat{\varepsilon}\left(\beta_{h}\right)$ and of the reference error $\left|\varepsilon\left(\beta_{h}\right)\right|$ shows a good behaviour of the estimation as the effectivity index $\rho$ is ranging from 1.04 to 1.23 .

In example 2, the reference failure probability is $P_{f \text {,ref }}=\Phi(-2.548)=5.5 \times 10^{-3}$. The bounds obtained by the FORM approximation are presented in Table VI. As in example 1 (see Table IV), the FEA leads to an underestimation of the failure probability when the mesh is not sufficiently refined. We obtain rapidly a good approximation of the upper bound of the failure probability with $P_{f, h}^{\text {upp }}=5.6 \times 10^{-3}$ on the second mesh (326 DoF), however, on this mesh the lower bound is very small $P_{f, h}^{\text {low }}=4.0 \times 10^{-3}$, and a reliability analysis with a more refined mesh is necessary to obtain less than $10 \%$ of uncertainty on the computation of failure probability with $P_{f}=5.2 \pm 0.3 \times 10^{-3}$ on the third mesh (670 DoF).

\section{CONCLUSIONS}

A method has been presented to recover lower and upper bounds for the computation of a reliability index in a finite element reliability analysis. This method is based on goal-oriented error estimation techniques that have been initially developed for deterministic computations. These bounds can be used to assess the quality of the finite element meshes used during a reliability analysis. They can also be used to derive bounds of the failure probability when a FORM approximation is used. Numerical experiments in linear elastic fracture mechanics show that the computed bounds are sufficiently sharp to control the error introduced by a finite element approximation in a reliability analysis. These bounds will allow to develop mesh adaptative strategy during a finite element reliability analysis.

\section{ACKNOWLEDGEMENTS}

This work was supported by the Publishing Arts Research Council under the grant number 98-1846389.

\section{REFERENCES}

1. Ditlevsen O, Madsen HO. Structural Reliability Methods. Wiley: New York, 1996.

2. Lemaire M. Fiabilité Des Structures. Hermes: Paris, 2005.

3. Hasofer AM, Lind NC. An exact and invariant first order reliability format. Journal of Engineering Mechanics (ASCE) 1974; 100(EM1):111-121.

4. Babuška I, Rheinboldt WC. A posteriori estimates for the finite element method. International Journal for Numerical Methods in Engineering 1978; 12:1597-1615. 
5. Ladevèze P, Leguillon D. Error estimate procedure in the finite element method and application. SIAM Journal on Numerical Analysis 1983; 20(3):485-509.

6. Zienkiewicz OC, Zhu JZ. A simple error estimator and adaptive procedure for practical engineering analysis. International Journal for Numerical Methods in Engineering 1987; 24:337-357.

7. Babuška I, Miller A. The post-processing approach of the finite element method. Part II: the calculation of the stress intensity factor. International Journal for Numerical Methods in Engineering 1982; 20:1111-1129.

8. Kelly DW, Isles JD. Procedures for residual equilibration and local error estimation in the finite element method. Communications in Numerical Methods in Engineering 1989; 5:497-505.

9. Rannacher R, Stuttmeier FT. A feedback approach to error control in finite element methods: application to linear elasticity. Computational Mechanics 1997; 19:434-446.

10. Peraire J, Patera A. Bounds for linear-functional outputs of coercive partial differential equations: local indicators and adaptive refinement. In Advances in Adaptive Computational Methods, Ladevèze P, Oden JT (eds). Elsevier: Amsterdam, 1998; 199-216.

11. Prudhomme S, Oden JT. On goal-oriented error estimation for elliptic problems: application to the control of pointwise errors. Computer Methods in Applied Mechanics and Engineering 1999; 176:313-331.

12. Ladevèze P, Rougeot P, Blanchard P, Moreau JP. Local error estimators for finite element analysis. Computer Methods in Applied Mechanics and Engineering 1999; 176:231-246.

13. Ohnimus S, Stein E, Walhorn E. Local error estimates of fem for displacements and stresses in linear elasticity by solving local neumann problems. International Journal for Numerical Methods in Engineering 2001; 52:727-746.

14. Prudhomme S, Oden JT, Westermann T, Bass J, Botkin ME. Practical methods for a posteriori error estimation in engineering applications. International Journal for Numerical Methods in Engineering 2003; 56:1193-1224.

15. Gallimard L, Panetier J. Error estimation of stress intensity factors for mixed-mode crack. International Journal for Numerical Methods in Engineering 2006; 68:299-316.

16. Gallimard L. Evaluation of the local quality of the von mises's stress and 12-norm of the stress. Engineering Computations 2006; 23(7/8):876-897.

17. Deb MK, Babuska I, Oden JT. Solution of stochastics partial differential equations using Galerkin finite element techniques. Computer Methods in Applied Mechanics and Engineering 2001; 190:6359-6372.

18. Ladeveze P, Florentin E. Verification of stochastic models in uncertain environments using the constitutive relation error method. Computer Methods in Applied Mechanics and Engineering 2006; 196:225-234.

19. Zhang Y, Der Kiureghian A. Two improved algorithms for reliability analysis. In Proceedings of the Sixth IFIP WG7.5 Reliability and Optimization of Structural System, Rackwitz R, Augusti G, Borri A (eds). Chapman \& Hall: London, 1994.

20. Wang L, Grandhi RV. Safety index calculation using intervening variables for structural reliability analysis. Computers and Structures 1996; 59(6):1139-1148.

21. Der Kiureghian A, Dakessian T. Multiple design points in first and second-order reliability. Structural Safety 1998; 20:37-49.

22. Mitteau JC. Error evaluation for the computation of failure probability in static structural reliability problems. Probabilistic Engineering Mechanics 1999; 14:115-119.

23. Mohamed A, Lemaire M, Mitteau JC, Meister E. Finite element and reliability: a method for compound variables-application on a cracked heating system. Nuclear Engineering and Design 1998; 185:185-202.

24. Pendola M, Mohamed A, Lemaire M, Hornet P. Combination of finite element and reliability methods in nonlinear fracture mechanics. Reliability Engineering and System Safety 2000; 70:15-27.

25. Rackwitz R, Fiessler B. Structural reliability under random load sequences. Computers and Structures 1979; 9(5):484-494.

26. Der Kiureghian A, de Stefano M. Efficients algorithms for second order reliability analysis. Journal of Engineering Mechanics 1991; 117(12):37-49.

27. Haukaas T, Der Kiureghian A. Strategies for finding the design point in non-linear finite element reliability analysis. Probabilistic Engineering Mechanics 2006; 21:133-147.

28. Strouboulis T, Babuşka I, Datta DK, Copps K, Gangaraj SK. A posteriori estimation and adaptive control of the error in the quantity of interest. Part 1: a posteriori estimation of the error in the von Mises stress and the stress intensity factor. Computer Methods in Applied Mechanics and Engineering 2000; 180:261-274.

29. Beckers R, Rannacher R. An optimal control approach to a posteriori error estimation in finite element method. Acta Numerica 2001; 19:1-102.

30. Heintz P, Samuelsson P. On adaptive strategies and error control in fracture mechanics. Computer and Structures 2004; 82:485-497.

31. Ruter M, Stein E. Goal-oriented a posteriori error in linear elastic fracture mechanics. Computer Methods in Applied Mechanics and Engineering 2006; 195:251-278.

32. Gallimard L. A constitutive relation error estimator based on traction-free recovery of the equilibrated stress. International Journal for Numerical Methods in Engineering 2009; 78:460-482.

33. Stern M, Becker EB, Dunham RS. A contour integral computation of mixed-mode stress intensity factors. International Journal of Fracture 1976; 12(3):359-368.

34. Tada H, Paris PC, Irwin GR. The Stress Analysis of Crack Handbook (2nd edn). Paris Inc.: St. Louis, MO, 1985. 